\title{
Routing Security in Wireless Sensor Networks
}

\author{
Hamid Shokrzadeh, M. R. Majma, A. Movassagh, and M. Saheb
}

\begin{abstract}
A sensor network consists of large number of sensor nodes that widely distributed in an environment and collect's the information of the environment. Necessarily the positioning of the sensor nodes, are not predetermined and not specified. This means that protocols and algorithms of sensor networks should be have Self-Organization capabilities. Another exclusive property of the sensor networks is collaboration capabilities and coordination among sensor nodes. The data must be transferred from the path that if it is not secure it can be cause Irreparable Injuries. In this article we will prepare about challenges of secure routing and will prepare a proposition to them.
\end{abstract}

Index Terms-Information, security, sensor networks, routing

\section{INTRODUCTION}

Wireless sensor networks have received significant recent attention in both the networking and operating systems communities. As wireless sensor networks have emerged as an exciting new research area in Computer Science, many of the logistical challenges facing those who wish to develop deploy, and debug applications on realistic large-scale sensor networks have gone unmet. Sensors generate an excessive amount of data which have been acquired from environmental information. A sensor network consists of large number of sensor nodes that widely distributed in an environment, and collect's the information of the environment. These networks are predicated on advances in miniaturization that will make it possible to design small form-factor devices with significant on-board computation, wireless communication, and sensing capabilities.Necessarily the positioning of the sensor nodes, are not predetermined and not specified. These properties make the possibility that place them into dangerous or inaccessible places. Other unique properties of the sensor networks, is capabilities of the collaboration and coordination among sensor nodes [1]-[3]. Each sensor node has a processor on its board, and instead of sending all of the raw data to the center or to the node that is responsible for processing the data and resulting from them, initially, it does a series of simple processing over the obtained data , and then it sends semi-processed data. Except of that each sensors has a little ability, combination of hundreds of small sensor offers

Manuscript received June 10, 2014; revised August 15, 2014. This work was supported in part by the Department of Computer Engineering, Pardis branch, Islamic Azad University.

H. Shokrzadeh and M. Saheb are with the Department of Computer Engineering, Pardis branch, Islamic Azad University, Pardis New City, Iran (e-mail: shokrzadeh@gmail.com, loverlifecode@gmail.com)

M. R. Majma was with Department of Computer Engineering, Pardis branch, Islamic Azad University, Pardis New City, Iran (e-mail: Majma@pardisiau.ac.ir)

A. Movassagh is with the Department of Electrical Engineering, Sharif University of Technology, Iran (e-mail: a.movassagh@gmail.com). new possibilities. Sensor itself has little ability to combine hundreds of small sensor offers new possibilities.

In fact, the power of wireless sensors networks are in the ability of using the large number of small nodes which can be assembled and self-organized and in the several cases such as simultaneously routing, environmental monitoring conditions, health monitoring of structures or equipment of the systems. The usage area of the sensor network applications are very extensive and include the applications such as agriculture, medicine, industrial and military applications. For example, one of the most common applications of this technology is remote environment monitoring [4]. As an example the leakage in a chemical factory in the large area of the factory can be automatically monitored by the hundreds of sensors that constructs the wireless network, and immediately notify to the center if any chemical leak is occurred or not. The wireless sensor network operates the monitoring and control of physical environments from the remote locations and does it within a good accuracy. The sensor nodes due to their low cost properties and there implement cases, have the computational and energetic limitations.

Fig. 1 shows an example of data flows in the sensor networks. In the several cases, the environment that should be monitored hasn't any energetic or communicational infrastructure, and it is essential that the sensor nodes protect themselves against low and limited source of energy, and maintained their relationship through a wireless communication channels. Despite the high-speed networking and computational capabilities of sensor networks, wide area applications, including aerospace, battlefields, medicine, robotics, weather and etc. are conceivable for such networks.

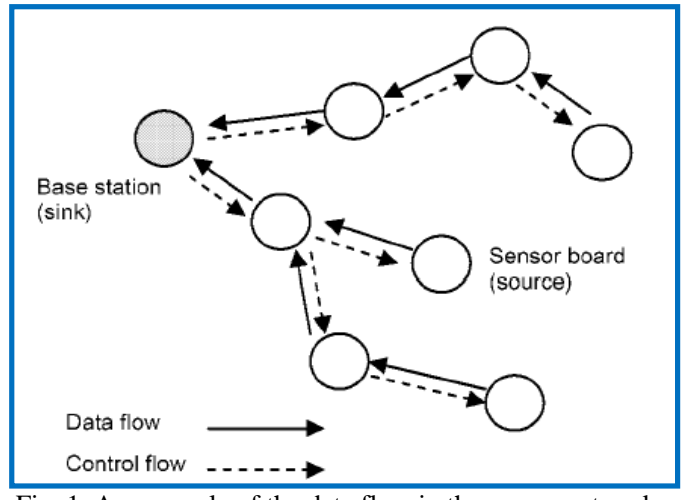

Fig. 1. An example of the data flow in the sensor networks.

Routing in order is used to receive a packet of data from a device and send it over the network to the other device on a different network.

Routers can be divided into the two main groups, software and hardware:

Hardware routers

The above mentioned routers, are hardware's that executes 
the specific software that produced by the manufacturers (currently they look just as black box). This software provides the routing capability to the router's that they can do the simplest and perhaps most important task of transferring data from one network to another network as well. Most of companies would prefer to use hardware routers because the hardware routers in the comparison of the software routers have high speed and increased reliability [4], [5].

\section{Software routers}

The software routers have a similar function with hardware routers and their main responsibility is to send data from one network to another network. A software router can be a NT server, or a Linux server. All proposed network operating systems, have the embedded routing capabilities:

\section{A. Shortest Path Algorithm}

The shortest path method is the simplest routing method. The main goal of this algorithm is to constitute the graph of the sub-network such that we suppose that the each node is a router and each edge is a communication line between two routers. In this case, each side will have a weight and with the regarding to the Dijkstra shortest path algorithm we can compute the possible shortest path.

\section{B. Distance Vector Algorithm}

In this way, the router is store a table (vector) in it, as a distance vector that store the best distance from every other routers place in the network. In this case, the better routing decisions are taken. This table is updated with the information of neighbor routers. Each item of the table elements has two parts, one of which shows the suitable output line to reaching to the desired router and the other is to estimate the timing interval of that router.

\section{Link State Routing}

The link-state routing idea is simple and presented in five sections, each router must be:

1) Discover the neighbors and know their network address.

2) Measure the delay or cost of the neighbors.

3) Create a package to keep the information obtained from neighbors.

4) To be send this packages to the all routers.

5) Compute the shortest path to any other path.

\section{Hierarchical Routing}

When hierarchical routing is used, the routers are divided to the regions that each router knows all the details of how packets sent to their destinations in his area, but they don't know anything about the internal structure of other regions. When several networks are connected together, it is normally that they must be considered as separate regions, until not need to the routers in a network, that aware about topology structure of other routers.

TinyOs is the first operating system that has been designed for wireless sensor networks [6]. Unlike to the many of operating systems, rather than multiple threading, TinyOs, is developed based on event-oriented programming model. Programs of TinyOs are composed of the event handler and the tasks. To writing TinyOs operating systems and developing the applications of it, using the specific programming language called nesC which is an extension of the $C$ programminglanguage.

\section{ROUTING AlgORITHMS BACKGROUND}

Here we present some of the security aware routing algorithm is wireless sensor network. These types of algorithms have been described by the concerned authors that the security of them would be promoted.

\section{A. TR Algorithm}

In this algorithm the topology of the network considered as a tree [7], [8]. Every potentially parent, has a special addressing space in the form of sub block that is used for assigning of the network addresses to the child's. Given the maximum of the child's of one parent $\left(\mathrm{C}_{\mathrm{m}}\right)$,maximum depth of the network $\left(\mathrm{L}_{\mathrm{m}}\right)$ and the maximum number of the router child's for a parent $\left(\mathrm{R}_{\mathrm{m}}\right)$, the size of the one address block that can be for each parent in the depth of $d(\operatorname{Cskip}(d))$, is calculated from the following formula:

$$
\operatorname{Cskip}(d)=\left\{\begin{array}{c}
1+c_{m}\left(l_{m}-d-1\right) r_{m}=1 \\
\frac{1+c m-r m-d_{m} r_{m} l_{m-d-1}}{1-r_{m}} \text { other wise }
\end{array}\right.
$$

Assigning the addresses to the nodes at depth $d$ : For the $k_{\text {th }}$ router:

$$
A_{K}=\left\{\begin{array}{l}
\text { Aparent }+\operatorname{Cskip}(d) \cdot(k-1)+1 \\
\text { Aparent }+\operatorname{Cskip}(d) \cdot R_{m}+k-R_{m}
\end{array}\right.
$$

For the $n_{\mathrm{th}}$ terminal stations:

$$
A_{n}=\text { Aparent }+C \operatorname{skip}(d) \cdot R_{m}+n \quad 1<=n<=c_{m}-R_{m}
$$

The $K_{\text {th }}$ router that has a positive $\operatorname{Cskip}(d)$ value can be distributing the address space between its child's.

If the $A_{m}$ be the address of source node in the depth of $d$ and $A_{d}$ be the address of destination node so the following relations are holds:

If $A_{m}<A_{d}<A_{m}+C \operatorname{skip}(d)$ then $A_{d}$ is the descendant of the $A_{m}$.

If $A_{d}>A_{m}+R_{m}$. Cskip $(d)$ then $A_{d}$ is the end child of the $A_{m}$.

Next step to reaching to the child, or descendants that are not the end utility's, is compute from the following formula:

$$
A_{x}=A_{m}+1+\left\lceil\frac{A_{d}-A_{m}-1}{\operatorname{Cskip}(d)}\right\rceil \cdot \operatorname{Cskip}(d)
$$

In the tree routing method, if the destination is a descendant, the device to be sent data's to the one of his children otherwise to be send's to his parent.

\section{B. Neighbors Table}

Each device, maintains a table of the neighbors that has all neighbors information's in it in the first step of transition [9].

If the users restrict the size of the neighbor table, the selected numbers of neighbor's entries are stored in the table. Contents of the neighbor entries are PAN properties, the additional address of the node, network address, device type and the type of relationship. It can be contains optionally, additional information such as the signal order (beacon), depth or permission of the links. The table entries are created when a node joins to the network.

\section{The CLZBRP Algorithm}

CLZRP provides solutions to maintain a list of neighbors. 
If the source wants to send the data to the destination, sends the data to his father, and the father node execute the formulas to find the destination from neighbors list. If it is near the neighborhood offers the data. As a result, we've reduced the delay time and save the energy and to increase the "throughput". The task of the routing layer is the finding the path from source to the destination. In the typical normal ad-hoc routing, when a source needs to send data to the destination via a broadcast message RREQ, RREP a path is created and then sent the data. In the CLZBRP instead of using of the cluster table from the network layer to recognize the address and the depth of the nodes, the resident path on the routing layer is deleted [8], [10].

\section{The IMPTR Algorithm}

This algorithm, follows the routing tree, but selecting the neighbor nodes as a next step node, occurs only if the path from neighboring node to the destination is shorter than when the TR protocol is used. ImpTR is discussed using the neighbor table, address schema with the same structure of networks and using the symmetric relations among the nodes. If the node $\mathrm{A}$ is a neighbor of the node $\mathrm{B}$, the node $\mathrm{A}$ is also neighbor of the node $\mathrm{B}$. Each node in the network contains a neighboring table. This table contains information such as the parent node, child nodes, private network identifier (identifier), MAC Address, network address, device type and communication. Normally this table of nodes generated in the joining process of searching the vicinity of itself to discovering its neighbors and to finding the perfect Father's to joining of it. This table is periodically updated by searching the neighbors [11].

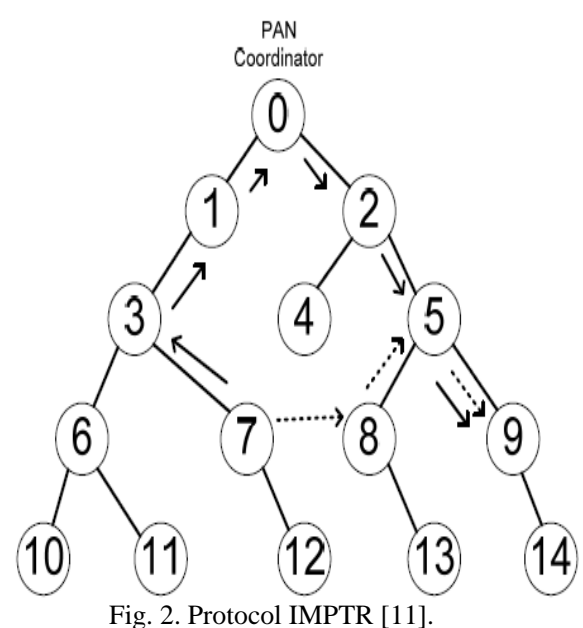

Configuration of the Network is shown in Fig. 2. The node 7 wants to send the data to the node 9. Each node has a neighbor table that contains information about neighbors within transmission range. Improving of this protocol consists of six stages. If the pointed address is the sink bode of the one of the descendants of the source node it's begin to checking it. If it existed, the data would be sent directly to the children. Otherwise, the following steps are performed.

Step 1: The source node checks that the sink node, is its father or not. This action is done by calculating of the father address using (1) and comparing it with the address of the sink.

SA the address of the source node and DA is the address of the sink node and $d$ is the depth of the source node.

$$
P A=S A+1+\left\lceil\frac{A_{d}-A_{m}-1}{\operatorname{Cskip}(d)}\right\rceil \cdot \operatorname{Cskip}(d)
$$

Step 2: The source node checks that the sink address whether is the one of its neighbors or not. If this was true the source node transmits the data packet to the related node.

Step 3: The source node checks that the sink address whether is the one of its neighbor's children or not. This is done by using of (2).

If this was true, first the packet is transmitted to the neighboring node. Since the neighbor node receives the packet, checks the packet of the algorithm and realized that the sink node is his child nodes. If more than one neighbor will satisfy the equation, the source node selects a node with the highest depth that is closer to the sink.

$N_{A}$ is the address of the source node.

$$
N_{A}<D_{A}<N_{A}+C \operatorname{skip}\left(d\left(N_{A}\right)-1\right)
$$

Step 4: The source node checks that the sink node whether is the one of the ancestors of neighbor nodes or not. This is done by calculating the addresses of neighboring nodes ancestor. Then it compares each ancestor with the sink node. It is clear that the coordinator of the ancestor of all nodes is common. $P_{N A(d)}$ is the address of the neighbor nodes parent with the depth of d. Each node has $P_{N A(0)}=0$.

Fig. 3 shows the algorithm of the computation of the ancestor address and comparing it with the sink node.

Step 5: The source node checks that the destination nodewhether is the node in the scope of the address of its neighbor's parent node or not. This is done by finding of the address of the neighbor's parent node by using (1). Equation 3 is then checked. If (3) was established, the source node will transmit the packets to its neighbor nodes. Then the neighboring nodes using the (1) is transferred the data packets to her father node. If all of the nodes are not acceptable then the parent node realizes that the source node is one of his underneath nodes. Fig. 2 shows this case in the data from of transmitting the data from node 7 to node 9 [12] and [8], [9].

$$
P_{N A}<D_{A}<P_{N A}+\operatorname{Cskip}\left(d\left(P_{N A}\right)-1\right)
$$

Step 6: If all the above steps may not be acceptable, the source node will transmits the packet to the parent node.

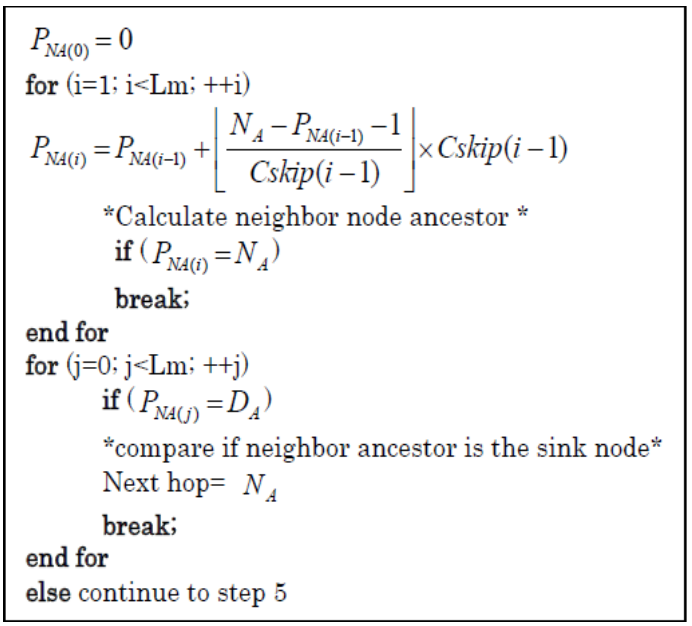

Fig. 3. Calculation of ancestor address. 


\section{SECURITY IN RouTING}

Most of the routing protocols of sensor networks are too simple, and in most cases it causes that, they are vulnerable to attacks. Most of the attacks against the network layer are happens because of the one of following reasons:

- Eavesdropping, modification and repeating routing information

- Optional Sending

- Sybil attacks

- Wormhole's

- HELLO flood attacks

In the following description pay attention to the difference between the attacks that try to directly modify the users' data, and attacks that try to substantial impacts on the routing topology [13], [14].

\section{A. Eavesdropping, Modification, or Repeating of Routing Information}

The most common direct attack against a routing protocol is the targeting of routing information that is exchanged between nodes. By eavesdropping, changing, or repeating of routing information, attackers can be exploited routing loops, creating mistake error messages, network segmentation, increasing the end-to-end delay, increase or shorten of source routes.

\section{B. Optional Sending}

The multi-step networks are working based on this consideration that, the innermost participating in routing nodes, are send the completely and intact the received messages to the next node. In selective attack, non-authorized node may not send specific messages to the next node and delete them, to ensure that these messages will not be published in any form. A simple form of this attack is like that the unauthorized node acts as a black hole and any package which is arrived to it does not send to the next node and delete it. If an attacker is threatened by its neighbor nodes it has to conclude that there should be elimination to the route, decides that search another way. A very smartest form of this attack is when an attacker sends packets selectively. An attacker tends that the packets originating from a specific number of nodes must be removed or changed and another packets to be sent correctly and this leads mistrust and suspicion towards the unauthorized acts of its decline [5], [15]. Selective send attacks are usually effective, when that the attacker stands trough on a path from a data stream. Although it is possible that an attacker eavesdrop the current passing route via its neighboring nodes and make jam or accident causing that the action such as selective sending to be simulated. Therefore, we believe that an attacker that attempting to do selective attack, certainly wants and tries to be trough the data stream and its current. In the next two sections we will discoursed about Sinkhole, and Sybil attacks, the tow mechanism that the attacker can effectively put itself over the desired data.

\section{Sinkhole Attacks}

In a sinkhole attacks, the attacker's goal is almost absorbed all traffics from the source node by a fall [11], [16], [17]. Because the nodes are in the path that the packets are passing through it, there are many opportunities that applications data can access in exposed. Sinkhole attacks can be a utility to the other attacks (such as a selective send) .Sinkhole attacks, happens usually by a node according to the routing algorithm that it will be more attractive than other nodes. For example, an attacker can eavesdrop or repeat an advertisement for a special path and quality route to the base station. Some of the protocols may evaluate the quality of a path with the end-to-end information that includes reliability information or quality or delayed information.

In this case, the attacker can handle a laptop with a powerful transmitter a way to create high quality by a high-power base station comes with a step, or by using a wormhole attack, which is discussed in the following section. It is likely that the attacker neighbor nodes to send their packets to the base station through an attacker node. The attacker can create a large sphere of influence, which absorbs all traffic towards the base station to all nodes that several steps are underway to unauthorized nodes. A sinkhole attack is a choice as a motive for the attack. Because of all the traffic flows through a node, an attacker can be selectively packages that are the source of all nodes in a certain area are removed or tampered with.

Wireless sensor networks are vulnerable to attacks that cause sinkhole due to their specific communication patterns. Since all packets have the same ultimate destination (in networks with only one base station), an unauthorized node only needs a high quality route to the base station to be able to penetrate to all the nodes.

\section{The Sybil Attack}

In a Sybil attack, an individual node offers several identification addresses to the other nodes in the network. The Sybil attacks can decrease the benefits of fault-toleration of distributed storages, dissemination and multi-path routing. Also Sybil attacks are a major threat to geographic routing protocols [18]. The routings such as geographic routing are often needs that the nodes exchange the coordination information with their neighbors to have efficient geographically routing and addressing of the path. A node in a network has only one fixed coordinates, but using the Sybil attacks an unauthorized node at the same time can be in more than one place.

\section{E. Selective Forwarding}

In this type of attacks one or more malicious nodes placed within the network and immediately after the receiving of information from its neighboring nodes, attempting to deflect or destroy the data, and finally, the data did not reach the main destination as well [15]. See Fig. 4.

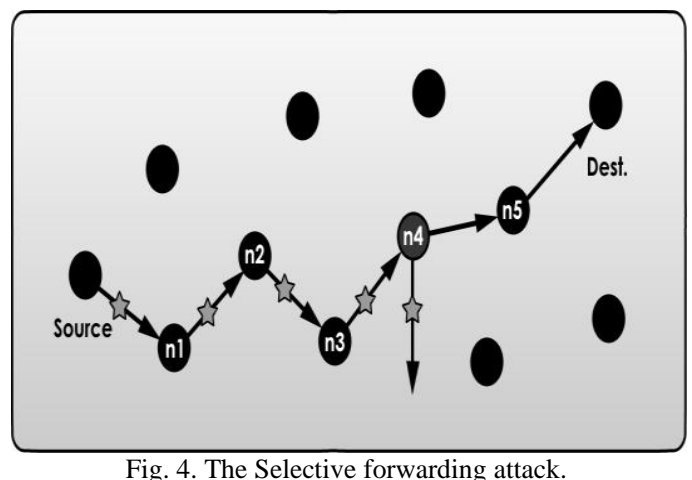

Attackavoiding approaches: 
- Using theshared keyand the encrypted data-to avoiding external attacks such as Sybil Attack and Selective Forwarding

- Assigning the unique key to the nodes and also restricting the neighbors' nodes to prevent Sybil Attacks.

- Careful designing of the routing protocol and using the geographical routingto avoiding Wormhole and Sinkhole attacks.

- Multi-directional routingand ensuring the neighbor nodes to avoid the Selective Forwarding attacks.

\section{CONCLUSION AND FUTURE WORK}

Secure routing is essential for widespread use of sensor networks in different applications, encryption and authentication of link layer, multi-path routing, validation of ID, bilateral links and authenticated broadcasting, can be protect the routing protocols against the aliens, forged routing information, Sybil attacks, HELLO floods and eavesdropping of information, and it is possible to equip the existing protocols, to this mechanisms. Sinkhole and wormhole attacks are brings up the fundamental challenges to design of routing protocols, And It is unlikely after thedesignof routing protocols thatdefense mechanisms against such attacks are exists. Designing of theprotocols against this tow attacks isdifficult. But with the protocols such asgeographic routing protocol we can hope topeace befound on the attacks.

\section{REFERENCES}

[1] P. Porkar and M. Gheisari, "Performance analysis of two sensor data storages," presented at European Conference on Complex Systems 2011 (ECCS11), Tenerife, Spain, 2011.

[2] A. Singla and R. Sachdeva, "Review on security issues and attacks in wireless sensor networks," International Journal of Advanced Research in Computer Science and Software Engineering, vol. 3, issue 4, April 2013.

[3] M. Chowdhury, M. F. Kader, and Asaduzzaman, "Security issues in wireless sensor networks: A survey," International Journal of Future Generation Communication and Networking, vol. 6, no. 5, pp. 97-116, 2013.

[4] D. Boyle and T. Newe, "Securing wireless sensor networks: Security architectures," Journal of Networks, vol. 3, no. 1, pp. 65-76, 2008

[5] X. Du and H. Chen, "Security in wireless sensor networks," in Proc. IEEE Wireless Communications, 2008.

[6] S. H. Hasan and A. G. A. Alquraishe, "Scalar multiplicationalgorithms for wireless sensor network," International Journal of Smart Home, October 2012.

[7] IEEE Standard for Information Technology Telecommunications and information exchange between systems LAN-MAN Specific requirements, "Wireless medium access control (MAC) and physical layer (PHY) specifications for low-rate wireless personal area network (LR-WPANs)," IEEE, 2003.

[8] W. Qui, E. Skafidas, and P. Hao, "Enhanced tree routing for wireless sensor networks," Ad Hoc Journal, vol. 7, issue 3, pp. 638-650, 2007.

[9] T. Kim, D. Kim, N. Park, S. Yoo, and T. S. Lopez, "Shortcut tree routing in ZigBee networks," in Proc. the Second International Symposium onWireless Pervasive Computing, pp. 42-47, 2007.

[10] P. Anitha and C. Chandrasekar, "Energy efficient routing algorithm for Zigbee using cross layer Zigbeebasedrouting (CLZBRP) protocol," International Journal of Computer Applications (IJCA), vol. 7, no. 3, pp. 18-21, 2011.

[11] C. Karlof and D. Wagner, "Secure routing in wireless sensor networks: Attacks and countermeasures," Tech. Report, University of California at Berkeley, 2009.

[12] M.-S. Pan and Y. Tseng, "Quick convergecast in ZigBee beacon-enabled tree-based wireless sensor networks," Journal of ComputerCommunications, vol. 31, issue 5, pp. 999-1011, 2007

[13] A. Zafar, A. Iqbal, and S. Lehri, "Mobile Ad Hoc network - A research perspective," presented at 6th National Conference on Computing for Nation Development, India, 2012.

[14] S. Sharma, "Energy-efficient secure routing in wireless sensor networks," Report of Dept of Computer Science and Engineering, National Institute of Technology Rourkela, Rourkela, Orissa, India, 2009.

[15] J. Granjal, R. Silva, and J. Silva, "Security in wireless sensor networks," Tech. Report: Centre for Informatics and Systems of the University of Coimbra, 2008

[16] E. Prem, K. Gilbert, B. Kaliaperumal, and E. B. Rajsingh, "Research issues inwirelesssensor network applications: A survey," International Journal of Information and Electronics Engineering, vol. 2, no. 5, pp. 702-706, September 2012.

[17] M. Sharifzadeh, S. Aragy, K. Bashash, S. Bashokian, and M. Gheisari, "A comparison with two semantic sensor data storages in total datatransmission," The International Journal of Soft Computing and Software Engineering, March 2013.

[18] T. Kim, D. Kim, N. Park, S. Yoo, and T. S. Lopez, "Shortcut tree routing innetworks," presented at the second International Symposium on Wireless Pervasive Computing, 2007.

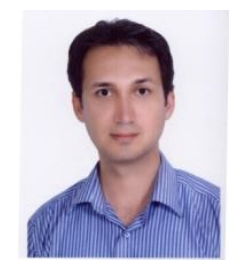

Hamid Shokrzadeh received the B.Sc. degree from Centeral Tehran branch, Islamic Azad University in computer hardware in 2004. Also he receievd his M.Sc. degree from Qazvin branch, Islamic Azad University in computer network in 2007. He received his $\mathrm{PhD}$ in computer architecture in Science and Research branch, Islamic Azad University of Thehran, Iran in 2013. His research interests include wireless sensor networks, routing algorithms and network simulations.

$\mathrm{He}$ is currently the faculty member of the Department of Computer Engineering, Pardis branch, Islamic Azad University, Iran since 2008. Also, $\mathrm{He}$ is the director of research office in this university since 2013.

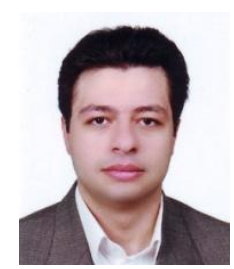

Mohammad Reza Majma is a Ph.D. student in Science and Research branch, Islamic Azad University located in Tehran, Iran. He received his MSc in IT engineering from Qazvin branch, Islamic Azad University, Iran in 2008. He is the faculty member of the Department of Computer Engineering, Pardis branch, Islamic Azad University since 2009. His research interests are wireless networks (sensor networks and ad hoc), modeling evaluation of computer networks, high performance computing and grid computing. 